\title{
Isotherm charts for material selection and method development with molecularly imprinted polymers and other sorbents
}

\author{
Zsanett Dorkó $^{1,2}$, Barbara Tamás ${ }^{1}$, George Horvai ${ }^{1,2, *}$ \\ ${ }^{1}$ Department of Inorganic and Analytical Chemistry, Budapest University of Technology and \\ Economics, Szent Gellert ter 4., H-1111 Budapest, Hungary \\ ${ }^{2}$ MTA-BME Research Group of Technical Analytical Chemistry, Szent Gellert ter 4., H-1111 \\ Budapest, Hungary \\ *Phone: +36-1-4631480; e-mail: george.horvai@mail.bme.hu
}

\begin{abstract}
A simple and efficient method is presented for assessing molecularly imprinted polymers (MIP) and other sorbents from the point of view of practical applications. The adsorption isotherms of the compounds, which need to be separated or detected in an application, are constructed from a small number of measured points on a log-log chart and then are compared graphically. Despite its simplicity and robustness this method reveals the information needed for optimal selection between MIPs and alternative sorbents. The design of separation or detection methods with MIPs is also supported by the proposed graphical isotherm comparison. Many experimental isotherms are presented supporting the proposed method.
\end{abstract}

Keywords: molecularly imprinted polymer, adsorption isotherm, polymer characterization, selectivity, propranolol, terbutylazine 


\section{INTRODUCTION}

Molecularly imprinted polymers (MIPs) are selective sorbents [1-4]. Molecular imprinting involves arranging polymerizable functional monomers around a template molecule, followed by polymerization and template removal. Therefore complementary size, shape, and functionalities toward the template in the polymer can be obtained. Consequently, MIPs are expected to rebind the template molecule and possibly also its structurally similar compounds selectively. Sometimes the template is actually chosen to be somewhat different from the real target compound of the planned application. This may have practical advantages.

As a control in each polymerisation, a non-imprinted polymer (NIP) is often synthesised in the same way but in absence of the template. The NIP and many other polymers, which are not made purposely selective towards a specific substance, may also display some sort of selectivity but this has not been much studied before.

MIPs have been used for the development of various analytical and technological methods such as liquid chromatography, capillary electrochromatography, solid-phase extraction (SPE), membrane separations, binding assays and sensors. The number of proposed industrial and environmental applications of MIPs has greatly increased in recent years [5-9]. Only in 2015 there were 61 patent applications published about MIPs worldwide [10].

It appears though, that the design principles which would allow the easy utilization of the vast amount of literature on MIPs for solving newly arising analytical and technical problems have not yet been developed. Reports on successful method development with MIPs usually appear to reflect a trial and error approach. Computer modelling at the molecular dynamics or quantum chemical level has proved useful in the design of particular MIPs [11-17] but this does not directly concern the problem discussed here.

It is the purpose of this paper to provide a simple tool which allows to select the most suited adsorbent (MIP or other material) for solving a practical problem. Another goal is to predict what problems and by which technology may be solved (or should not be solved because simpler and cheaper alternatives exist) with a particular MIP. The novel tool is a simple experimental and graphical method. It will be presented using two kinds of widely researched MIPs, prepared for beta blockers and triazines, respectively. The application of the proposed 
method is not limited to MIPs and it will be applied here also to other, alternative sorbents, like commercial ion exchangers.

The structure of the remaining part of this paper is as follows. First, the proposed graphical isotherm comparison method is presented and its advantages over alternative methods are explained. Next, the main technical and analytical applications of MIPs are assessed using typical examples of the isotherm charts. Finally the possibilities of replacing MIPs by cheaper and more easily available sorbents are presented on the basis of the respective isotherm charts.

\section{MATERIALS AND METHODS}

\subsection{MATERIALS}

Methacrylic acid (MAA), ethylene glycol dimethacrylate (EDMA), propranolol hydrochloride, dibenzylamine (DBA), $(R)$-(-)-2-benzylamino-1-phenylethanol (Rbz), terbutylazine, prometryn, acetic acid (glacial, $\mathrm{AcOH}$ ), benzoic acid were purchased from Sigma. Azobisisobutyronitrile (AIBN) was ordered from Fluka. $\mathrm{HCl}$ and atrazine were obtained from Riedel-De Haën. $N$-benzylbenzamide and benzophenone were ordered from Alfa Aesar. Acetonitrile, methanol, acetic acid (HPLC grade) and diethylamine were ordered from Merck. Ketoprofen was a generous gift of the Hungarian National Institute of Pharmacy and Nutrition. Dimethylformamide (DMF) was acquired from Reanal. Water was purified with a Milli Q Direct 8system. Weak cation exchanger (non-imprinted SPE sorbent, Chromabond, HR-XCW) was also studied.

\subsection{INSTRUMENTATION}

The following instruments were used: Grant-Bio PTR-35 multi-rotator, Eppendorf Minispin centrifuge, Perkin Elmer Series 200 HPLC, Purospher RP18-e $(125 \times 3$ mm, $5 \mu \mathrm{m}$, Merck) reversed phase column, ZymarkTurboVap LV evaporator.

\subsection{POLYMER PREPARATION}

Polymers were prepared as described previously, using the method of Andersson [18] for propranolol MIP and the method of Ferrer et al. [19] for terbutylazine MIP synthesis. The corresponding non-imprinted polymers (NIPs) were also prepared in the same manner but without the addition of template. 
All further experimental details (including the composition and coding of the polymers used) can be found in the Supplementary Material.

\section{RESULTS AND DISCUSSION}

\subsection{THEORY}

\subsubsection{The need for isotherm measurements in MIP characterization}

Any novel MIP needs to be characterized before it can be meaningfully used. There exist many characterization methods like porosity measurements, infrared spectroscopy, thermal analysis and others, but the most important feature to be measured is the adsorption isotherm of the MIP [20]. The isotherm tells how much of a given substance is bound (adsorbed) by the MIP from solutions of different concentrations in equilibrium. More accurately the isotherm shows the equilibrium bound concentration ( $q$ in $\mathrm{mol} / \mathrm{kg}$ ) as a function of the equilibrium solution concentration ( $c$ in $\mathrm{M}$ ). This function is independent of the phase ratio employed in the measurement.

In many reports on MIPs no isotherm is measured but other, apparently similar methods are used to prove the efficiency of imprinting or the selectivity of the MIP. Such methods include elution chromatographic measurements on a MIP HPLC column, measurement of the selectivity of a MIP based sensor or simply demonstration of the successful solution of a practical problem with the MIP. None of these methods is inherently sufficient to characterize the MIP in such a way that the results might be transferred to other applications or even to slightly changed conditions. It has been shown for example [21] that HPLC selectivity between two compounds depends on apparently irrelevant factors as the length and diameter of the MIP HPLC column. A further advantage of the isotherm method is its independence from adsorption kinetics. The latter may need to be measured separately for certain applications.

For the characterization of a MIP one needs actually more than one isotherm, even at a single temperature. Most important is the isotherm of the target substance, i.e., the substance which needs to be separated or detected by the intended application. The isotherms of other substances which may also be adsorbed from the matrix handled in a practical problem are also relevant, either as interferences or as additional targets. In many papers only two 
isotherms, those of the target compound on the MIP and on the NIP, respectively, are presented.

Isotherms are typically measured either with frontal chromatography or with batch equilibrium experiments. Frontal chromatography is a very precise technique, it can provide precision and accuracy of ca. $1 \%$ in the measurement of $q[22,23]$. However, in many cases, such highly precise measurements are not possible, because the polymer may not be suitable for high quality HPLC column packing (the amount, size, shape or pressure resistance of the particles are not appropriate) or it is too time consuming to do many frontal chromatographic experiments.

Therefore many authors publish isotherms measured with about 2-10\% uncertainty, typically by batch (static) equilibration. This is a very simple method, which needs a lower amount of polymer, less time and experience than frontal chromatography. It will be shown in this paper that such measurements can be appropriate for practical polymer testing. However, because of their moderate accuracy, the usual isotherm evaluation methods should not be used with them. One such widely used method for isotherm evaluation is the fitting of various isotherm equations to the measured data. This method should only be employed if the isotherms are very precisely measured in a rather wide concentration range [24]. Furthermore, the fitted parameters of the commonly used isotherm equations do not provide directly useful information for technical applications or even only for judging the selectivity of adsorption. These problems indicate that there is a need for a robust method, which can help chemists in choosing the appropriate polymer for a given separation or detection task. Fast and cheap methods are in demand, which allow using moderately precise data, collected only in the limited concentration range defined by the expected practical application.

\subsubsection{The $\log c-\log q$ isotherm plot and its advantages}

In this paper, a new way for evaluating MIP and NIP isotherm data is demonstrated. The equilibrium adsorbed concentration $(q)$ is plotted against the equilibrium solution concentration $(c)$ of the respective compounds in a log-log plot. Two or more such log-log isotherm plots are visually compared to obtain the necessary information for polymer selection or method design.

Examples below will show that when plotting and comparing isotherms on log-log plots: 
1. it is sufficient to do isotherm measurements only in the expected range of the practical application

2. 2-3 points per log concentration unit are satisfactory

3. isotherms need not be measured with high precision.

The $\log -\log$ isotherm plots of MIPs and of some alternative sorbents are often close to linear in a certain concentration range, at least in a rough approximation. A linear trend line is helpful for visual comparison, but does not necessarily implicate Freundlich type behaviour. Many other authors have also plotted the measured MIP isotherms on log-log plots [25-28], but this was done because it was assumed, that the Freundlich isotherm equation fits the experimental data, and the authors wanted to obtain the Freundlich parameters. In the present work Freundlich or any other isotherm equation fitting is not attempted. Instead, efforts are made to find the advantages of $\log$ - $\log$ plots directly. As will be seen, a simple visual observation of the isotherm points in the log-log plot is very informative.

As noted earlier, different isotherms of interest may be measured and plotted. For example the isotherms of the target compound (which is often also the template used in MIP synthesis) on the NIP and MIP, respectively, or the isotherms of potential interferents (which may be the chemical analogues of the target) can be measured. Comparison of the respective $\log c-\log q$ plots allows directly the determination of the commonly used parameters like distribution ratio $(D)$, imprinting factor $(I F)$ and selectivity $(\mathrm{Sel})$, (and their concentration dependence, which has been often neglected) as Fig. 1 shows:

1. Distribution ratio, $D=q / c$ : the vertical distance of the isotherm from the $\log q=\log c$ line at any $c$ value gives the logarithm of $D$ at the particular $c$.

2. Imprinting factor, IF: the vertical distance between the isotherms of NIP and MIP, respectively, gives the logarithm of the $I F$ at any given $c$.

3. Selectivity, Sel: the vertical distance between the isotherms of the target compound and an analogue of it or of an (other) interferent, respectively, on the MIP gives the logarithm of $\mathrm{Sel}$ at any given $c$.

\section{Fig. 1}

Reading the logarithm of distribution ratio $(D)$, imprinting factor $(I F)$ and selectivity (Sel) from the log-log plot. The inset shows the respective isotherms on a lin-lin plot. 
Note that the same isotherms, when plotted on a linear plot (inset of Fig. 1), require more effort to obtain $\mathrm{D}, \mathrm{Sel}$ and $I F$ and their concentration dependences. Particularly, the visual impression about these quantities, as obtained from the linear isotherms, may be misleading unless the linear isotherm is plotted on several different scales as for example in [29].

In this paper, isotherms of different compounds are measured on various polymers, and these isotherms are compared on log-log plots. On the plots the equilibrium bound concentration $(q)$ is shown in $\mathrm{mol} / \mathrm{kg}$, and the equilibrium solution concentration $(c)$ is given in $\mathrm{mol} / \mathrm{L}$. The mass of polymer has been measured in the air dry state. The reproducibility of the measurements was typically $2.5 \%$ of the measured concentration values.

The use of $\log c-\log q$ plots extends beyond polymer characterization. In later sections of this paper it will be shown how polymers can be selected for particular applications with the help of the $\log -\log$ plot.

\subsection{APPliCATION OF THE $\log c-\log q$ PlOtS TO ASSESS SELECTIVITY AND TO ASSIST METHOD SELECTION}

\subsubsection{The selectivity of molecularly imprinted polymers assessed by $\log c-\log q$ plots}

As a demonstration of the use of $\log c-\log q$ plots the selectivity of a propranolol MIP toward different other secondary amines, and the selectivity of a terbutylazine MIP toward different other triazines have been investigated, see Fig. 2. Isotherms were measured in batch binding experiments in the respective porogenic solvents (the solvent in which the imprinting occurred), because in some cases it was shown that the optimal rebinding conditions require the same, or very similar solvent as used for polymerization [30-35].

Fig. 2

MIP selectivity ( $\mathrm{Sel}$ ) and its concentration dependence (isotherms were measured in batch binding experiments)

a, Isotherms of secondary amines on propranolol MIP (P1) in the porogenic solvent $(\mathrm{ACN})$

b, Isotherms of triazines on terbutylazine MIP (P4) in the porogenic solvent (toluene) 


\section{For names and structures of analytes and polymers see the Supplementary Material.}

Fig. 2 reflects, that all isotherms are in a rough approximation linear on log-log plots (in the tested concentration range), and their slopes are lower than 0.6. This means, that the isotherms are non-linear in a $c-q$ plot (see inset of Fig. 1), and the distribution ratio of the compounds is changing with the concentration. Interestingly, isotherms of different secondary amines are almost parallel in Fig. 2a (i.e., propranolol MIP (P1) in acetonitrile). On the terbutylazine MIP (P4) the isotherms of terbutylazine and atrazine are almost coinciding (Fig. 2b). This is not surprising, as the difference between these two chlorotriazine molecules is minor. However, adsorption of prometryn (a thiotriazine compound) at low concentrations is less than the adsorption of the two chlorotriazines, and the respective isotherm lines diverge. It has been reported indeed, that chlorotriazine MIPs exhibit pronounced selectivity for their chlorotriazine templates compared to thiotriazines [19, 36-38].

In general, log-log isotherms of similar compounds may be nearly parallel (as seen for different secondary amines on the propranolol MIP) or they may quite definitely diverge when going to lower concentrations (like terbutylazine vs prometryn on the terbutylazine MIP).

A further important observation from Fig. 2 is that the selectivities even between not very closely similar compounds, like propranolol and DBA, are moderate. The value of log $\mathrm{Sel}$ is at no concentration (in the measured range) more than 1, i.e., Sel is less than 10. A selectivity value between 1 and 10 is very useful in some applications but may be insufficient in others. In the present case the selectivity between propranolol and some other, moderately strong secondary nitrogen bases, is moderate. On the other hand, as Fig. 3a shows, adsorption of many compounds not related to propranolol (terbutylazine, benzophenone, ketoprofen, benzoic acid, $N$-benzylbenzamide) is negligible on the propranolol MIP (P1) in ACN. Thus the selectivity of the propranolol MIP against these compounds is very high.

Fig. 3

Adsorption of different compounds on propranolol MIP (P1) and on NIP (P2)

(In each case $10 \mathrm{mg}$ polymer+ $1 \mathrm{~mL} 5 * 10^{-4} \mathrm{M}$ solution in $\mathrm{ACN}$ ) 
The selectivity of a MIP between two similar compounds need not always be low, either. The selectivity of the terbutylazine MIP between two similar compounds, terbutylazine and prometryn, may become quite high at concentrations lower than studied here, since the respective isotherms are so divergent (Fig. 2b).

Note here that Fig. 2a and $\mathbf{2 b}$ relate to measurements in the respective porogens because, as mentioned above, the porogen is often the optimal solvent for rebinding (perhaps due to a high fidelity of the binding sites if the imprinting and rebinding medium are the same). Nevertheless in numerous cases MIP selectivity against closely related compounds (e.g., between enantiomeric pairs) could be improved by optimizing the composition of the rebinding solvent $[39,40]$. Such optimization might always be usefully rounded up by comparing the log-log isotherm plots in the porogen and in the optimal rebinding medium, respectively.

The selectivity of MIPs has often been characterized by other measures than the one used here, e.g., by the imprinting factor or by the recovery obtained in solid-phase extraction with the MIP. Both have very complicated relationship with the simple, thermodynamic selectivity measure used in this paper. They are often misleading and certainly unsuitable for being used in systematic method design. It can happen for instance, that the IF is high, but the difference between the MIP and the NIP is small in the practical application [41]. Note that the difficulty of adequately defining selectivity is generally observed in analytical chemistry [42, 43].

\subsubsection{Using the log-log isotherms to understand and design practical} applications

In this section some of the usual applications of MIPs will be discussed. The general observations made about the MIP isotherms in the previous section will be used to assess the useful ranges of MIP applications.

The main applications of MIPs are as follows:

- HPLC on MIP columns [39, 44-46] 
- Solid phase extraction (SPE or MISPE) $[47,48]$

- Membrane separations [49-51]

- Sensors [52-54]

- Binding assays [55-57]

- Selective removal of low concentration contaminants in industrial or environmental technologies $[5,8,9]$

In all of these applications selective separation or detection are needed. If selectivity between widely different compounds or groups of compounds is required, MIPs are suitable for any of the above tasks. However, as the examples above have shown, the selectivity of MIPs between similar compounds is often quite limited. It appears that the vertical distance between the logarithmic isotherms of two similar compounds on a MIP (i.e., the selectivity between these compounds) is typically low except at concentrations below micromolar, or unless a suitable solvent medium (different from the porogen) can be found, where selectivity is much improved [39, 40]. Below this concentration level selective binding assays [58-61] and selective sensing $[62,63]$ have been indeed successful even against interferents similar to the target compound. At higher concentrations, however, separation of similar compounds by MIPs may mostly require chromatography, or in technological applications cascades of separation units. In sensing, individual sensors may not be sufficiently selective for a particular compound in this higher concentration range, but sensor arrays [64, 65], consisting of a set of differently imprinted MIPs, may prove to be useful.

There exist many practical problems where the target compound or a group of chemically similar target compounds needs to be separated or distinguished from other, unrelated compounds. This is often the case with solid phase extraction. MIPs are very useful here as Fig. 3a had shown. However, this task may eventually also be solved by less sophisticated adsorbents as Fig 3b shows. Claims, that the success of MISPE is due to the selectivity achieved by imprinting, require more scrutiny. MISPE is essentially a low efficiency chromatographic separation. As such it is hardly suitable to separate compounds for which the selectivity of the MIP is less than 10 . Therefore it is likely that the frequently reported success of MISPE as a sample pretreatment for HPLC is due to the absence from the samples of (chromatographic) interferents closely related to the target compound(s). The same situation may also prevail with some successful MIP sensor applications and with the successful removal of trace environmental or industrial contaminants by MIPs. 
On the other hand, MIPs and NIPs appear to be underutilized at high solution concentrations or more correctly at high polymer loadings $(q)$. Isotherm studies at such high loadings appear to be rare although they might prove to be useful. For example it has been found [66] that a propranolol imprinted MIP can actually bind propranolol equivalent to more than $25 \%$ of its $\mathrm{COOH}$ content, while the binding capacity of a very similarly prepared MIP had been estimated from the binding isotherm (measured at lower concentrations) as merely $4 \%$ of its $\mathrm{COOH}$ content [18]. (The somewhat different experimental conditions between these experiments do not seem to support so much discrepancy.) On the other hand, as Fig. 2a had shown, the selectivity between similar compounds may be preserved even when the MIP is loaded close to its maximum binding capacity. Note here that isotherms on a linear scale, such as in the inset of Fig. 1, do not allow to judge by inspection that the selectivity is retained with increasing concentration. The comparison of the logarithmic isotherms in Fig. 1 and Fig. 2 satisfies this requirement. 


\subsection{SELECTION OF ALTERNATIVES TO REPLACE MIPS}

In this section a series of examples will be shown for finding convenient replacements for MIPs by analysing the $\log c-\log q$ isotherm plots of different adsorbents.

\subsubsection{Replacement of the MIP by non-imprinted polymers}

In many MIP applications it is demonstrated experimentally that the NIP is inferior to the MIP. This apparent inferiority of the NIP may be due, however, to using it in a method optimized for the MIP, not for the NIP. Indeed, NIPs themselves are interesting for some applications [67, 68] or have comparable performance to MIPs [69-72]. Usually they have their own selectivity [73-77] and during the imprinting process, this selectivity and/or their adsorption ability is only slightly improved (if at all). These data from the literature make it likely that MIPs can occasionally be replaced by NIPs in practical applications. In other studies commercial sorbents made without the use of imprinting were found to exhibit similar practical performance to MIPs [69, 78, 79].

Inspection of logarithmic isotherms can explain such empirical results. Fig. 4 compares the isotherms of propranolol and DBA measured in ACN on the propranolol MIP, on its NIP and on a commercial, conventional cation exchanger, respectively.

\section{Fig. 4}

\section{Propranolol and DBA isotherms on propranolol MIP, on the NIP and on a commercial weak cation exchanger (ion ex.) in $\mathrm{ACN}$}

Fig. 4 shows, that, surprising as it may be, the NIP has the highest selectivity, but also a commercial generic weak cation exchanger is able to differentiate these two compounds. So in this example, imprinting is not needed for separation. The MIP may be preferred to the NIP at low concentrations for the higher level of binding on it, but not for its selectivity. At higher concentrations even this advantage of this MIP against the NIP is greatly reduced. The commercial cation exchanger performs even better. It shows much higher binding than the MIP at any concentration, and it displays only slightly worse selectivity. 
In view of these results MIPs can be advantageously replaced by a NIP or a commercial sorbent (not made by imprinting) even if the separation of (moderately) similar compounds is needed. When selectivity between chemically more different compounds is needed, the NIP does not lag behind the MIP, either. This is shown by Fig. 3b.

Whether the replacement of the MIP by a NIP is useful may depend on the particular medium where the adsorbent will be used. It has been shown above that in acetonitrile and at relatively high concentrations the NIP can replace the MIP. In other media, for example in ACN modified with acetic acid, this may not be true, as shown by Fig. S2. The selectivity of the MIP is only slightly reduced by acetic acid while the selectivity of the NIP (data not shown) completely disappears in ACN modified with acetic acid. Note that such medium effects on selectivity are easily detected with the $\log c-\log q$ plots but would be hard to note otherwise.

A special case of replacing MIPs with NIPs could be the use of NIPs made in solvents different from the one used for imprinting the MIP. Fig. 5 shows that a NIP prepared in the highly polar DMF (which solvent is not eminently suitable for imprinting) has similar properties at high concentrations to the propranolol MIP which had been prepared in ACN. The selectivity of this NIP (distance between the stars) even surpasses that of the MIP (distance between the circles), just like had been observed with the NIP prepared in ACN (distance between the squares). The latter, i.e., the usual NIP binds, however, less propranolol than the NIP made in DMF. This means that NIPs prepared under slightly modified conditions (compared to the conditions which are optimal for imprinting) may advantageously replace MIPs in real applications.

Fig. 5

\section{Isotherms of propranolol and DBA on propranolol MIP (P1) and two NIPs (P2 and P5) made in different solvents (ACN and DMF, respectively)}

\subsubsection{Imprinting with other templates than the target compound}

Since in technical applications MIPs in large quantities are needed, the cost of purchasing a suitable MIP, made with the target compound as the template, may be prohibitive. In analytical applications a drawback of MIPs made with the target compound as template is the 
slow bleeding of the template. This has often been eliminated by imprinting with a close analogue of the target. In the present work imprinting with an only very distantly similar but simple and cheap template has been tested to replace the target compound propranolol in the imprinting. This template has been diethylamine. Success of this method has been expected based on the results of Kotova et al. [80] and Legido-Quigley and coworkers [81]. The diethylamine MIP has very similar binding (including selectivity) properties as the propranolol MIP (see Fig. S3 in the Supplementary Material, solid symbols). This result shows that even a remotely similar compound may be suitable to replace the target compound in imprinting and ease the problems with costs and/or with bleeding.

The usefulness of these results is supported by noting that a commercial producer of MIPs solves practical analytical or technological problems not by making a new MIP with the compound in question as the template, but rather by screening their inventory of MIPs for suitability in solving the actual problem [82]. Their screening platform is commercially available, and it is based on 96-well plates with a MIP library consisting of different functional monomers. The apparent success of this approach supports the conclusion that MIPs (and NIPs) are useful novel polymeric adsorption materials for solving many practical problems, but there is little reason to make a new MIP for every new problem. It is very likely that a limited number of well selected MIPs and NIPs can be sufficient for solving the majority of real life problems.

\section{CONCLUSIONS}

In this paper a new way for comparing MIP isotherms has been presented. The equilibrium adsorbed concentration $(q)$ was plotted against the equilibrium solution concentration $(c)$ in a $\log -\log$ plot. Simple visual comparison of such plots has been found adequate for judging the suitability of a MIP for practical (technological, analytical, etc.) applications. The plots can also help to find alternatives of MIPs like the same kind of polymer prepared without imprinting or a polymer imprinted with a cheap alternative compound, or even traditional, commercially available polymers.

While applied here to a new generation of adsorbents, the MIPs, the method introduced in this paper is equally useful to characterize other adsorbents as well. Examples of this have been 
shown here when the method was applied to non-imprinted polymers, including a commercial ion exchanger.

The proposed graphical isotherm assessment method appears to be preferable to isotherm equation fitting. Equation fitting, when correctly done, is not only technically very demanding and time consuming, but the judgement of selectivity from isotherm equations is also quite circumstantial. With the here proposed simple method the characterization of MIPs by much fewer experimental data is possible. Precision of the measured data is also less critical than in equation fitting.

The examples presented in this paper relate to non-covalent imprinting with small molecules (MW<ca. 1000). The here introduced isotherm charting method can certainly be applied to other imprinting methods and to imprinting with macromolecules, but the relationship between the isotherms of MIPs made by those methods needs further experimental studies. Interactions involving two simultaneously adsorbed substances also require further studies.

\section{Acknowledgements}

The financial support of OTKA, Hungary (Grant No. K120075) is gratefully acknowledged. Z. Dorkó acknowledges the support of the Ernő Pungor Scholarship.

\section{References}

[1] B.Sellergren (Ed.), Molecularly Imprinted Polymers. Man-Made Mimics of Antibodies and Their Application in Analytical Chemistry; Techniques and Instrumentation in Analytical Chemistry, first ed., Elsevier Science, Amsterdam, 2001.

[2] K. Haupt (Ed.), Molecular Imprinting, Springer, Heidelberg, 2012.

[3] C. Alvarez-Lorenzo, A. Concheiro (Eds.), Handbook of Molecularly Imprinted Polymers, first ed., Smithers Rapra, Shrewsbury, 2013.

[4] M.J. Whitcombe, N. Kirsch, I.A. Nicholls, Molecular imprinting science and technology: a survey of the literature for the years 2004-2011, J. Mol. Recognit. 27 (2014) 297-401.

[5] G. Szekely, M.C.A. de Sousa, M. Gil, F.C. Ferreira, W. Heggie, Genotoxic impurities in pharmaceutical manufacturing: Sources, regulations, and mitigation, Chem. Rev. 115 (2015) 81828229.

[6] B. Sellergren, P. Manesiotis, A. Hall, Molecularly imprinted polymers for extraction of components from foodstuffs US 2006/0292545. 2006 Dec 28.

[7] B. Sellergren, E. Fritz, G. Szekely, Polymers for drug purification. WO 2012/172075. 2012 December 20.

[8] D.L. Huang, R.Z. Wang, Y.G. Liu, G.M. Zeng, C. Lai, P. Xu, B.A. Lu, J.J. Xu, C. Wang, C. Huang, Application of molecularly imprinted polymers in wastewater treatment: a review, Environ. Sci. Pollut. Res. 22 (2015) 963-977.

[9] C.P. Silva, M. Otero, V. Esteves, Processes for the elimination of estrogenic steroid hormones from water: a review, Environ. Pollut. 165 (2012) 38-58.

[10] MIPdatabase, Patents in 2015. http://www.mipdatabase.com/patents.php?year=2015 , 2016 (accessed 26. 05. 16) 
[11] S.A. Piletsky, K. Karim, E.V. Piletska, C.J. Day, K.W. Freebairn, C. Legge, A.P.F. Turner, Recognition of ephedrine enantiomers by molecularly imprinted polymers designed using a computational approach, Analyst 126 (2001) 1826-1830.

[12] T. Muhammad, L. Cui, J.D. Wang, E.V. Piletska, A.R. Guerreiro, S.A. Piletsky, Rational design and synthesis of water-compatible molecularly imprinted polymers for selective solid phase extraction of amiodarone, Anal. Chim. Acta 709 (2012) 98-104.

[13] I.A. Nicholls, H.S. Andersson, C. Charlton, H. Henschel, B.C.G. Karlsson, J.G. Karlsson, J. O'Mahony, A.M. Rosengren, K.J. Rosengren, S. Wikman, Theoretical and computational strategies for rational molecularly imprinted polymer design, Biosens. Bioelectron. 25 (2009) 543-552.

[14] I.A. Nicholls, H.S. Andersson, K. Golker, H. Henschel, B.C.G. Karlsson, G.D. Olsson, A.M. Rosengren, S. Shoravi, S. Suriyanarayanan, J.G. Wiklander, S. Wikman, Rational design of biomimetic molecularly imprinted materials: theoretical and computational strategies for guiding nanoscale structured polymer development, Anal. Bioanal. Chem. 400 (2011) 1771-1786.

[15] D. Cleland, G.D. Olsson, B.C. Karlsson, I.A. Nicholls, A. McCluskey, Molecular dynamics approaches to the design and synthesis of PCB targeting molecularly imprinted polymers: interference to monomer-template interactions in imprinting of 1,2,3-trichlorobenzene, Org. Biomol. Chem. 12 (2014) 844-853.

[16] L.S. Fernandes, P. Homem-de-Mello, E.C. de Lima, K.M. Honorio, Rational design of molecularly imprinted polymers for recognition of cannabinoids: A structure-property relationship study, Eur. Polym. J. 71 (2015) 364-371.

[17] W.M. Yang, L.K. Liu, X.N. Ni, W. Zhou, W.H. Huang, H. Liu, W.Z. Xu, Computer-aided design and synthesis of magnetic molecularly imprinted polymers with high selectivity for the removal of phenol from water, J. Sep. Sci. 39 (2016) 503-517.

[18] L.I. Andersson, Application of molecular imprinting to the development of aqueous buffer and organic solvent based radioligand binding assays for (S)-propranolol, Anal. Chem. 68 (1996) 111-117.

[19] I. Ferrer, F. Lanza, A. Tolokan, V. Horvath, B. Sellergren, G. Horvai, D. Barcelo, Selective trace enrichment of chlorotriazine pesticides from natural waters and sediment samples using terbuthylazine molecularly imprinted polymers, Anal. Chem. 72 (2000) 3934-3941.

[20] R.J. Ansell, Characterization of the binding properties of molecularly imprinted polymers, Adv. Biochem. Eng. Biotechnol. 150 (2015) 51-93.

[21] B. Toth, T. Pap, V. Horvath, G. Horvai, Which molecularly imprinted polymer is better?, Anal. Chim. Acta 591 (2007) 17-21.

[22] F. Gritti, G. Guiochon, Limits of the numerical estimation of the adsorption energy distribution from adsorption isotherm data using the expectation-maximization method, J. Chromatogr. A 1144 (2007) 208-220.

[23] F. Kamarei, F. Gritti, G. Guiochon, J. Burchell, Accurate measurements of frontal analysis for the determination of adsorption isotherms in supercritical fluid chromatography, J. Chromatogr. A 1329 (2014) 71-77.

[24] G. Guiochon, A. Felinger, D.G. Shirazi, A.M. Katti, Fundamentals of Preparative and Nonlinear Chromatography, second ed., Academic Press, 2006, Boston.

[25] H. Kim, D.A. Spivak, New insight into modeling non-covalently imprinted polymers, J. Am. Chem. Soc. 125 (2003) 11269-11275.

[26] J.J. Torres, N. Gsponer, C.L. Ramirez, D.M.A. Vera, H.A. Montejano, C.A. Chesta, Experimental and theoretical studies on the enantioselectivity of molecularly imprinted polymers prepared with a chiral functional monomer, J. Chromatogr. A 1266 (2012) 24-33.

[27] R.J. Umpleby, S.C. Baxter, M. Bode, J.K. Berch, R.N. Shah, K.D. Shimizu, Application of the Freundlich adsorption isotherm in the characterization of molecularly imprinted polymers, Anal. Chim. Acta 435 (2001) 35-42.

[28] M. Arabi, A. Ostovan, M. Ghaedi, M.K. Purkait, Novel strategy for synthesis of magnetic dummy molecularly imprinted nanoparticles based on functionalized silica as an efficient sorbent for the determination of acrylamide in potato chips: Optimization by experimental design methodology, Talanta 154 (2016) 526-532.

[29] H. Kim, G. Guiochon, Adsorption on molecularly imprinted polymers of structural analogues of a template. Single-component adsorption isotherm data, Anal. Chem. 77 (2005) 6415-6425. 
[30] F. Meier, B. Schott, D. Riedel, B. Mizaikoff, Computational and experimental study on the influence of the porogen on the selectivity of 4-nitrophenol molecularly imprinted polymers, Anal. Chim. Acta 744 (2012) 68-74.

[31] D. Spivak, M.A. Gilmore, K.J. Shea, Evaluation of binding and origins of specificity of 9ethyladenine imprinted polymers, J. Am. Chem. Soc. 119 (1997) 4388-4393.

[32] H.J. Kim, G. Guiochon, Thermodynamic studies of the solvent effects in chromatography on molecularly imprinted polymers. 3. Nature of the organic mobile phase, Anal. Chem. 77 (2005) 24962504.

[33] C. Baggiani, L. Anfossi, P. Baravalle, C. Giovannoli, C. Tozzi, Selectivity features of molecularly imprinted polymers recognising the carbamate group, Anal. Chim. Acta 531 (2005) 199-207.

[34] C. Yu, K. Mosbach, Influence of mobile phase composition and cross-linking density on the enantiomeric recognition properties of molecularly imprinted polymers, J. Chromatogr. A 888 (2000) 63-72.

[35] D.A. Spivak, K.J. Shea, Investigation into the scope and limitations of molecular imprinting with DNA molecules, Anal. Chim. Acta 435 (2001) 65-74.

[36] V. Horvath, B. Lorantfy, B. Toth, J. Bognar, K. Laszlo, G. Horvai, Preparation of terbutylazine imprinted polymer microspheres using viscous polymerization solvents, J. Sep. Sci. 32 (2009) $3347-$ 3358.

[37] F.G. Tamayo, M.M. Titirici, A. Martin-Esteban, B. Sellergren, Synthesis and evaluation of new propazine-imprinted polymer formats for use as stationary phases in liquid chromatography, Anal. Chim. Acta 542 (2005) 38-46.

[38] F. Chapuis, V. Pichon, F. Lanza, B. Sellergren, M.C. Hennion, Retention mechanism of analytes in the solid-phase extraction process using molecularly imprinted polymers. Application to the extraction of triazines from complex matrices, J. Chromatogr. B 804 (2004) 93-101.

[39] R.J. Ansell, Molecularly imprinted polymers for the enantioseparation of chiral drugs, Adv. Drug Delivery Rev. 57 (2005) 1809-1835.

[40] H. Kim, G. Guiochon, Thermodynamic studies on the solvent effects in chromatography on molecularly imprinted polymers. 1. Nature of the organic modifier, Anal. Chem. 77 (2005) 1708-1717. [41] X.M. Jiang, W. Tian, C.D. Zhao, H.X. Zhang, M.C. Liu, A novel sol-gel-material prepared by a surface imprinting technique for the selective solid-phase extraction of bisphenol A, Talanta 72 (2007) 119-125.

[42] Z. Dorko, T. Verbic, G. Horvai, Selectivity in analytical chemistry: two interpretations for univariate methods, Talanta 132 (2015) 680-684.

[43] Z. Dorko, T. Verbic, G. Horvai, Comparison of the single channel and multichannel (multivariate) concepts of selectivity in analytical chemistry, Talanta 139 (2015) 40-49.

[44] E. Turiel, A. Martin-Esteban, Molecularly imprinted polymers: towards highly selective stationary phases in liquid chromatography and capillary electrophoresis, Anal. Bioanal. Chem. 378 (2004) 1876-1886.

[45] J. Haginaka, Monodispersed, molecularly imprinted polymers as affinity-based chromatography media, J. Chromatogr. B 866 (2008) 3-13.

[46] S. Pardeshi, S.K. Singh, Precipitation polymerization: a versatile tool for preparing molecularly imprinted polymer beads for chromatography applications, RSC Adv. 6 (2016) 23525-23536.

[47] A. Beltran, F. Borrull, P.A.G. Cormack, R.M. Marce, Molecularly-imprinted polymers: useful sorbents for selective extractions, Trends Anal. Chem. 29 (2010) 1363-1375.

[48] A. Martin-Esteban, Molecularly-imprinted polymers as a versatile, highly selective tool in sample preparation, Trends Anal. Chem. 45 (2013) 169-181.

[49] M. Ulbricht, Membrane separations using molecularly imprinted polymers, J. Chromatogr. B 804 (2004) 113-125.

[50] S.A. Piletsky, T.L. Panasyuk, E.V. Piletskaya, I.A. Nicholls, M. Ulbricht, Receptor and transport properties of imprinted polymer membranes - a review, J. Membr. Sci. 157 (1999) 263-278.

[51] F. Trotta, M. Biasizzo, F. Caldera, Molecularly imprinted membranes, Membranes (Basel) 2 (2012) 440-477.

[52] M.C. Moreno-Bondi, F. Navarro-Villoslada, E. Benito-Pena, J.L. Urraca, Molecularly imprinted polymers as selective recognition elements in optical sensing, Curr. Anal. Chem. 4 (2008) 316-340. 
[53] M.J. Whitcombe, I. Chianella, L. Larcombe, S.A. Piletsky, J. Noble, R. Porter, A. Horgan, The rational development of molecularly imprinted polymer-based sensors for protein detection, Chem. Soc. Rev. 40 (2011) 1547-1571.

[54] S. Li, Y. Ge, S.A. Piletsky, J. Lunec (Eds.), Molecularly Imprinted Sensors: Overview and Applications, first edition ed., Elsevier Science, Oxford, 2012.

[55] L.I. Andersson, Molecular imprinting for drug bioanalysis - A review on the application of imprinted polymers to solid-phase extraction and binding assay, J. Chromatogr. B 739 (2000) 163 173.

[56] N. Lavignac, C.J. Allender, K.R. Brain, Current status of molecularly imprinted polymers as alternatives to antibodies in sorbent assays, Anal. Chim. Acta 510 (2004) 139-145.

[57] Y. Ge, A.P. Turner, Molecularly imprinted sorbent assays: recent developments and applications, Chemistry 15 (2009) 8100-8107.

[58] L.I. Andersson, R. Muller, G. Vlatakis, K. Mosbach, Mimics of the binding-sites of opioid receptors obtained by molecular imprinting of enkephalin and morphine, Proc. Natl. Acad. Sci. U. S. A. 92 (1995) 4788-4792.

[59] E. Benito-Pena, M.C. Moreno-Bondi, S. Aparicio, G. Orellana, J. Cederfur, M. Kempe, Molecular engineering of fluorescent penicillins for molecularly imprinted polymer assays, Anal. Chem. 78 (2006) 2019-2027.

[60] B.T.S. Bui, A.S. Belmont, H. Witters, K. Haupt, Molecular recognition of endocrine disruptors by synthetic and natural 17 beta-estradiol receptors: a comparative study, Anal. Bioanal. Chem. 390 (2008) 2081-2088.

[61] G. Vlatakis, L.I. Andersson, R. Muller, K. Mosbach, Drug assay using antibody mimics made by molecular imprinting, Nature 361 (1993) 645-647.

[62] T. Alizadeh, Graphene/graphite/molecularly imprinted polymer nanocomposite as the highly selective gas sensor for nitrobenzene vapor recognition, J. Environ. Chem. Eng. 2 (2014) 1514-1526.

[63] V.K. Gupta, M.L. Yola, N. Atar, A novel molecular imprinted nanosensor based quartz crystal microbalance for determination of kaempferol, Sens. Actuators, B 194 (2014) 79-85.

[64] T. Hirsch, H. Kettenberger, O.S. Wolfbeis, V.M. Mirsky, A simple strategy for preparation of sensor arrays: molecularly structured monolayers as recognition elements, Chem. Commun. (2003) 432-433.

[65] K.D. Shimizu, C.J. Stephenson, Molecularly imprinted polymer sensor arrays, Curr. Opin. Chem. Biol. 14 (2010) 743-750.

[66] Z. Dorko, A. Szakolczai, T. Verbic, G. Horvai, Binding capacity of molecularly imprinted polymers and their non-imprinted analogs, J. Sep. Sci. 38 (2015) 4240-4247.

[67] E. Piletska, K. Karim, R. Coker, S. Piletsky, Development of the custom polymeric materials specific for aflatoxin B1 and ochratoxin A for application with the ToxiQuant T1 sensor tool, J. Chromatogr. A 1217 (2010) 2543-2547.

[68] E. Piletska, G. Stavroulakis, K. Karim, M. Whitcombe, I. Chianella, A. Sharma, K. Eboigbodin, G. Robinson, S. Piletsky, Attenuation of Vibrio fischeri quorum sensing using rationally designed polymers, Biomacromolecules 11 (2010) 975-980.

[69] A. Guerreiro, A. Soares, E. Piletska, B. Mattiasson, S. Piletsky, Preliminary evaluation of new polymer matrix for solid-phase extraction of nonylphenol from water samples, Anal. Chim. Acta 612 (2008) 99-104.

[70] N.M. Maier, G. Buttinger, S. Welhartizki, E. Gavioli, W. Lindner, Molecularly imprinted polymer-assisted sample clean-up of ochratoxin A from red wine: merits and limitations, J. Chromatogr. B 804 (2004) 103-111.

[71] S. Zorita, B. Boyd, S. Jonsson, E. Yilmaz, C. Svensson, L. Mathiasson, S. Bergstrom, Selective determination of acidic pharmaceuticals in wastewater using molecularly imprinted solid-phase extraction, Anal. Chim. Acta 626 (2008) 147-154.

[72] M. Pascale, A. De Girolamo, A. Visconti, N. Magan, I. Chianella, E.V. Piletska, S.A. Piletsky, Use of itaconic acid-based polymers for solid-phase extraction of deoxynivalenol and application to pasta analysis, Anal. Chim. Acta 609 (2008) 131-138.

[73] J.D. Dai, J.S. He, A.T. Xie, L. Gao, J.M. Pan, X. Chen, Z.P. Zhou, X. Wei, Y.S. Yan, Novel pitaya-inspired well-defined core-shell nanospheres with ultrathin surface imprinted nanofilm from 
magnetic mesoporous nanosilica for highly efficient chloramphenicol removal, Chem. Eng. J. 284 (2016) 812-822.

[74] Z.F. Xu, L. Liu, Q.Y. Deng, Study on the mechanism of binding specificity of metoclopramideimprinted polymers, J. Pharm. Biomed. Anal. 41 (2006) 701-706.

[75] M. Li, R. Li, J. Tan, Z.T. Jiang, Titania-based molecularly imprinted polymer for sulfonic acid dyes prepared by sol-gel method, Talanta 107 (2013) 203-210.

[76] J.X. Guo, Y.Z. Wang, Y.J. Liu, C.J. Zhang, Y.G. Zhou, Magnetic-graphene based molecularly imprinted polymer nano-composite for the recognition of bovine hemoglobin, Talanta 144 (2015) 411419.

[77] Q.C. Zhang, X.H. Xiao, G.K. Li, Porous molecularly imprinted monolithic capillary column for on-line extraction coupled to high-performance liquid chromatography for trace analysis of antimicrobials in food samples, Talanta 123 (2014) 63-70.

[78] W. Boonjob, H. Sklenarova, F.J. Lara, A.M. Garcia-Campana, P. Solich, Retention and selectivity of basic drugs on solid-phase extraction sorbents: application to direct determination of beta-blockers in urine, Anal. Bioanal. Chem. 406 (2014) 4207-4215.

[79] B. Du, T.T. Qu, Z. Chen, X.H. Cao, S.P. Han, G.P. Shen, L. Wang, A novel restricted access material combined to molecularly imprinted polymers for selective solid-phase extraction and high performance liquid chromatography determination of 2-methoxyestradiol in plasma samples, Talanta 129 (2014) 465-472.

[80] K. Kotova, M. Hussain, G. Mustafa, P.A. Lieberzeit, MIP sensors on the way to biotech applications: Targeting selectivity, Sens. Actuators, B 189 (2013) 199-202.

[81] C. Legido-Quigley, J. Oxelbark, E. De Lorenzi, A. Zurutuza-Elorza, P.A. Cormack, Chromatographic characterisation, under highly aqueous conditions, of a molecularly imprinted polymer binding the herbicide 2,4-dichlorophenoxyacetic acid, Anal. Chim. Acta 591 (2007) 22-28.

[82] R. Kecili, J. Billing, D. Nivhede, B. Sellergren, A. Rees, E. Yilmaz, Fast identification of selective resins for removal of genotoxic aminopyridine impurities via screening of molecularly imprinted polymer libraries, J. Chromatogr. A 1339 (2014) 65-72. 